\title{
Efficacy and safety of rituximab for systemic lupus erythematosus treatment: a meta-analysis
}

\author{
Shanshan Wu*1, Yanhai Wang*2, Jiaojiao Zhang ${ }^{3}$, Bo Han ${ }^{1}$, Baishan Wang ${ }^{1}$, Wanli Gao ${ }^{1}$, \\ Ning Zhang ${ }^{1}$, Cheng Zhang ${ }^{1}$, Feng Yan ${ }^{1}$, Zhijing Li ${ }^{1}$
}

1. Clinical Laboratory Department, The Affiliated Hospital of Liaoning University of Traditional Chinese Medicine, No. 33 Beiling Street, Huanggu District, Shenyang, 110032, Liaoning, China.

2. Clinical Laboratory Department, Dong Sheng People's Hospital of Ordos, Ordos, 017000, The Inner Mongolia Autonomous Region, China.

3. Clinical Laboratory Department, The Fourth Affiliated Hospital of China Medical University, No. 4 Chongshan Road, Huanggu District, Shenyang, 110032, Liaoning, China.

* These authors contributed equally to this work.

\begin{abstract}
:
Background: Given the inconsistency of previous studies and the newly emerging evidence, we decided to conduct a meta-analysis.

Methods: The meta-analysis included 2 randomized controlled trials and 13 observational studies 742 patients in total. Qualified studies were properly searched from databases. Data were analyzed by the RevMan 5.3 software. Results were demonstrated as WMD , SMD and RR with $95 \%$ CIs, $I^{2}$ and $\mathrm{P}$ value.

Results: we observed that a remarkable increase of complement C3 in the rituximab group than placebo group (WMDfixed $=7.67 \mathrm{mg} / \mathrm{dL}, 95 \% \mathrm{CIs}=-0.16 \sim 15.50, \mathrm{I} 2=0 \%, \mathrm{P}=0.05)$. A significant increase of complement $\mathrm{C} 4$ was observed in the rituximab group than placebo group (WMDfixed $=3.14 \mathrm{mg} / \mathrm{dL}, 95 \% \mathrm{CIs}=1.06 \sim 5.22, \mathrm{I}^{2}=0 \%, \mathrm{P}=0.003$ ). Notably decreased peripheral $\mathrm{CD} 19+\mathrm{B}$ cells in rituximab group than placebo group (WMDfixed $=-117.93 \mathrm{n} / \mu 1,95 \% \mathrm{CI}=-172.94 \sim-62.91$, $\left.\mathrm{I}^{2}=0 \%, \mathrm{P}<0.0001\right)$ in RCTs. Patients with severe or refractory SLE got more satisfactory efficacy results after receiving rituximab in observational studies, such as British Isles Lupus Assessment Group index score, SLE Disease Activity Index score, complement C3/C4, anti-dsDNA antibodies, peripheral CD19 ${ }^{+} \mathrm{B}$ cells and so on. Safety profiles were no difference between rituximab and placebo groups.

Conclusion: although the efficacy of rituximab is highly controversial for SLE, our study shows that rituximab presents a satisfying efficacy and safety for SLE.
\end{abstract}

Keywords: Efficacy; safety; rituximab; systemic lupus erythematosus; meta-analysis.

DOI: https://doi.org/10.4314/ahs.v20i2.41

Cite as: Wu S, Wang Y, Zhang J, Han B, Wang B, Gao W, et al. Efficacy and safety of rituximab for systemic lupus erythematosus treatment: a meta-analysis. Afri Health Sci. 2020; 20(2): 871-884. https:// doi.org/10.4314/abs.v20i2.41

\section{Introduction}

Systemic lupus erythematosus (SLE) is an auto-immune

\section{Corresponding author: \\ Shanshan Wu, \\ Clinical Laboratory Department, \\ The Affiliated Hospital of Liaoning \\ University of Traditional Chinese Medicine, \\ No. 33 Beiling Street, Huanggu District, \\ Shenyang, 110032, Liaoning, China. \\ Email: wuma19811982@163.com}

disease that involves widely differing tissues and organs with diverse clinical symptoms. The incidence of SLE in women is estimated to be approximately 10 times higher than that in men ${ }^{1}$. However, the pathogenesis of SLE is still unclear; the production of autoantibodies and deposition of immune complexes in multiple organs leads to various abnormalities, including rash, arthritis, serositis, cytopenia, nephritis, and psychosis ${ }^{2,3}$. Conventional therapies for SLE include nonsteroidal anti-inflammatory drugs, corticosteroids, hydroxychloroquine (HCQ) and immunosuppressive agents. Among these therapies, corticosteroids and immuno- 
suppressive agents are primarily associatedwith mortality and morbidity ${ }^{4}$. More effective treatments should be developed for SLE. B cells are widely thought to play a crucial role in the pathogenesis of SLE. B cells act as antigen-presenting cells and present autoantigens to T cells; subsequently, $\mathrm{T}$ cells activate and produce cytokines. $T$ cell cytokines stimulate and induce B cells to secrete autoantibodies. Autoantigen-specific B cells interact with $\mathrm{T}$ cells and produce autoantibodies that are present only in non-healthy individuals. The evidence suggests that depletion of $\mathrm{B}$ cells has a favorable effect on $\mathrm{SLE}^{3}$. Rituximab is a chimeric monoclonal antibody that targets the CD20 marker ${ }^{5}$. Findings of previous studies have suggested that rituximab has a beneficial effect and satisfactory tolerance profile for serious refractory $\mathrm{SLE}^{6-8}$. However, two randomized placebo-controlled double-blinded trials showed no clinically significant differences between rituximab and a placebo,10. These previous studies are controversial. Borba found unsatisfactory variations between rituximab and a placebo in the efficacy results of a systematic review and meta-analysis, which included results for clinical reactions, British Isles Lupus Assessment Group (BILAG) C scores, time-adjusted area under the curve minus baseline(AUCMB) for the BILAG index, and modification of the SF-36 physical component summary(PCS $)^{11}$. Given the inconsistency of previous studies and newly emerging evidence, we decided to conduct a meta-analysis. The purpose of our study is to determine other parameters to investigate the efficacy and safety of rituximab for SLE patients that may be used for reference by clinicians.

\section{Methods}

We conducted a meta-analysis to estimate the efficacy and safety of rituximab treatment for SLE and followed the Cochrane Handbook ${ }^{12}$.

\section{Inclusion and exclusion criteria}

The inclusion criteria were as follows: (1) The SLE diagnosis satisfied the standards specified by the American College of Rheumatology13. (2) The trials included rituximab as an intervention treatment for SLE. (3) Placebo group as control group in RCTs. Baseline group when patients did not receive rituximab as control group in observational studies. (4) The study included efficacy and safety results, and the parameters of effi- cacy were the BILAG score, SLEDAI score, comple-ment C3/ C4 levels, anti-dsDNA antibodies, peripheral $\mathrm{CD} 19^{+} \mathrm{B}$ cells, serum creatinine, $24-\mathrm{h}$ urinary protein and $\mathrm{Up} /$ Ucr. The safety results included the incidence of SAEs, deaths, infections, gastrointestinal disorders, infusion-related SAEs and infusion-related AEs. (5) Both RCT and observational studies that met the above conditions can be included in this study. Trials without clinical outcomes and articles that were merely obtaina- ble as abstracts were excluded from the meta-analysis11. No language restrictions were implemented.

\section{Search strategy and data extraction}

The PubMed, Cochrane Library, EMBASE, Clinicaltrials and CNKI and Chinese database of WanFang databases were searched for relevant articles, most of which were published in English. The search was conducted using the following strategy, according to recognized methodologies ${ }^{45}$. Descriptors in the PubMed database included the Medical Subject Headings terms "Lupus Erythematosus, Systemic"and "Rituximab" combined with free terms. The process showed the results of electronic searches with Boolean operators such as "AND" and "OR". Two reviewers (SSW and JJZ) independently performed electronic searches on several databases. Initial screening was performed by title and abstract. Then, two reviewers read the full-text article during the final screening. In the case of discrepancies between the two reviewers, the results were discussed with a third reviewer. Reviewers assessed the included studies according to the Cochrane Collaborations tool; the evaluation bias risk is reported in the Cochrane Handbook ${ }^{46}$. Two reviewers independently extracted data, and other reviewers verified and ensured that data had been exactly recorded. When data could not be obtained from the full-text article, we contacted the authors by e-mail to obtain raw data.

\section{Quality assessment}

The quality of included RCTs was estimated by the Jadad scale, which ranges from 0 to 5 . Low-quality RCTs frequently receive a score of 2 or less, and high-quality research receives a score of at least $3^{47}$. According to the Cochrane Collaboration approach, the risk of bias is reported as low, moderate, or high; reporting of bias leads to an uncertain potential risk of bias. The quality of the included observational studies was estimated by the Newcastle-Ottaa Scale (NOS) ${ }^{48}$. The NOS score for studies ranges from 5 to 9 . Scores $\geq 6$ are defined as high-quality research. Thirteen observational studies were defined as high-quality, and the average score was 7.5, as shown in Table 4.

\section{Data analysis}

The extracted data are expressed as the means \pm SD at 
baseline and at the endpoint. The results were reported as weighted mean differences (WMDs), standard mean differences (SMDs) and relative risks (RRs) with 95\% CIs, $\mathrm{I}^{2}$ values and $\mathrm{P}$ values. The $\mathrm{I}^{2}$ value indicated the heterogeneity among included studies; $\mathrm{I}^{2}$ values of over $25 \%, 50 \%$, and $75 \%$ are commonly defined as low, medium and high heterogeneity, respectively ${ }^{49}$. When $\mathrm{I}^{2} \geq 50 \%$, heterogeneity is significant, the random effect model is applied. In this case, the inverse variance statistical method was utilized to calculate the WMD or SMD with $95 \% \mathrm{CI}$. The RR and 95\% CI were calculated with the Mantel-Haenszel statistical method. A value of $\mathrm{I}^{2} \leq 25 \%$ was regarded, as low heterogeneity, and the fixed-effects model was utilized. To ensure the homogeneity of the included studies, when $\mathrm{I}^{2} \geq 75 \%$, a study with obvious heterogeneity would be removed to determine whether it was the source of heterogeneity. All tests were two-tailed, and a value of $\mathrm{P} \leq 0.05$ was re-

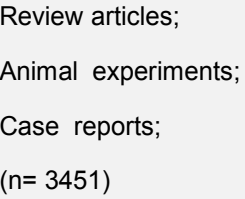

Fig.1 Flowchart of study selection

Inherent differences exist between RCTs and observational studies; therefore, they were analyzed separately. RCTs exhibited high quality and low risk of bias. Observational studies with greater numbers of patients garded as a significant difference. The statistical analysis was performed using RevMan version 5.3.

\section{Results}

\section{Review profiles and included studies}

We retrieved 4139 articles in the following electronic databases: PubMed 631, Cochrane Library 12, EMBASE 3465, Clinicaltrials 4, China National Knowledge Infrastructure (CNKI) 9 and Chinese database of WanFang 18. After duplicates were removed ( $\mathrm{n}=650), 3451$ articles were deemed unsuitable according to their title or abstract because animal experiments were conducted or the studies were case reports, meeting abstracts or reviews. The remaining 38 articles were assessed independently after a full-text reading by two reviewers (SSW and JJZ). At the end of the screening, 2 RCTs and 13 observational studies were included based on the established inclusion criteria ${ }^{9,10,12-24}$. A flowchart of the literature search and screening procedure is shown in Fig. 1. were included, but the potential risk for selection bias and residual confounding were increased. The baseline features of the 2 included RCTs are summarized in Table 1 . The baseline characteristics of the 13 observational studies are shown in Table 2. 


\begin{tabular}{|c|c|c|c|c|c|c|c|c|c|c|c|c|c|c|}
\hline \multicolumn{2}{|c|}{ Study } & \multirow{2}{*}{$\begin{array}{c}\text { Jadad } \\
\text { Score } \\
3 \\
\end{array}$} & \multirow{2}{*}{$\begin{array}{l}\mathrm{N}^{*} \\
72 \\
\end{array}$} & \multirow{2}{*}{\begin{tabular}{l}
\multicolumn{1}{|c}{ Enrolled patients } \\
Patients with class III \\
or classIV lupus \\
nephritis
\end{tabular}} & \multirow{2}{*}{$\begin{array}{c}\begin{array}{c}\text { Endpoint } \\
\text { (week) }\end{array} \\
52\end{array}$} & \multirow{2}{*}{$\begin{array}{c}\begin{array}{c}\text { Age } \\
\text { (year) } \\
\text { mean } \pm \text { SD }\end{array} \\
31.8 \pm 9.6\end{array}$} & \multirow{2}{*}{$\begin{array}{r}\begin{array}{r}\text { Female } \\
\text { sex }(\%)\end{array} \\
87.5 \\
\end{array}$} & \multirow{2}{*}{$\begin{array}{c}\begin{array}{c}\text { SLEduration } \\
\text { (year) } \\
\text { mean } \pm \mathrm{SD}\end{array} \\
32.4 \pm 48.0 \text { (months) }\end{array}$} & \multirow{2}{*}{\begin{tabular}{l}
\multicolumn{1}{c}{ Treatment } \\
\\
Rituximab or \\
placebo $1,000 \mathrm{mg}$ \\
administered \\
intravenously on \\
days $1,15,168$ and \\
182
\end{tabular}} & \multirow{2}{*}{$\begin{array}{c}\begin{array}{c}\text { BILAG score } \\
\text { mean } \pm \text { SD }\end{array} \\
15.3 \pm 6.4\end{array}$} & \multirow{2}{*}{$\begin{array}{c}\begin{array}{c}\text { Anti-dsDNA } \\
\text { (IU/mL) } \\
\text { mean } \pm \text { SD }\end{array} \\
453.2 \pm 795.7\end{array}$} & \multirow{2}{*}{$\begin{array}{c}\mathrm{C} 3 \\
(\mathrm{mg} / \mathrm{dL}) \\
\text { mean } \pm \mathrm{SD} \\
73.6 \pm 29.4 \\
\end{array}$} & \multirow{2}{*}{$\begin{array}{c}\mathrm{C} 4 \\
(\mathrm{mg} / \mathrm{dL}) \\
\text { mean } \pm \mathrm{SD} \\
14.7 \pm 8.5\end{array}$} & \multirow{2}{*}{$\begin{array}{c}\begin{array}{c}\text { CD19-B cells }(\mathrm{n} / \mu \mathrm{L} \\
\text { mean } \mathrm{SD}\end{array} \\
280.5 \pm 350.2\end{array}$} \\
\hline LUNAR & rituximab & & & & & & & & & & & & & \\
\hline & placebo & & 72 & & & $29.4 \pm 9.3$ & 93.1 & $28.8 \pm 51.6$ (months) & & $15.3 \pm 6.2$ & $350.6 \pm 634.0$ & $74.1 \pm 27.9$ & $13.8 \pm 9.4$ & $243.2 \pm 313.5$ \\
\hline EXPLORER & rituximab & 3 & 169 & $\begin{array}{l}\text { Patients with } \\
\text { moderately to severely } \\
\text { active extrarenal SLE }\end{array}$ & 52 & $40.2 \pm 11.4$ & 89.9 & $8.5 \pm 7.2$ & $\begin{array}{l}\text { Rituximabor placebo } \\
(2 \text { infusions } \\
1,000 \mathrm{mg} \text { given } 14 \\
\text { days } \\
\text { apart)administered } \\
\text { intravenously on } \\
\text { days } 1,15,168 \text { and } \\
182\end{array}$ & $14.0 \pm 5.1$ & $282.3 \pm 799.0$ & $99.0 \pm 32.3$ & $15.6 \pm 8.1$ & $210.4 \pm 286.1$ \\
\hline & placebo & & 88 & & & $40.5 \pm 12.8$ & 93.2 & $8.7 \pm 7.6$ & & $14.5 \pm 5.6$ & $209.2 \pm 535.2$ & $96.3 \pm 35.3$ & $15.5 \pm 8.6$ & $182.8 \pm 196.1$ \\
\hline
\end{tabular}

Table 2 Safety of rituximab $(1,000 \mathrm{mg})$ at week 52

\begin{tabular}{|c|c|c|c|c|c|}
\hline Outcome & $\begin{array}{l}\text { Rituximab } \\
\end{array}$ & Placebo & RR(95\%CIs) & $\mathrm{I}^{\mathrm{I}(\%)}$ & $\mathrm{P}$ \\
\hline $\begin{array}{l}\text { Severe adverse events } \\
\text { nats }\end{array}$ & 88 & 61 & $0.94[0.72,1.23]$ & 0 & 0.67 \\
\hline Deaths & 6 & 1 & $2.86[0.51,16.15]$ & 0 & 0.23 \\
\hline Infections & 30 & 29 & $0.73[0.46,1.16]$ & 28 & 0.18 \\
\hline Gastrointestinal disorders & 11 & 13 & $0.55[0.25,1.22]$ & 0 & 0.14 \\
\hline \multicolumn{6}{|l|}{ Any infusion-related severe adverse events } \\
\hline & 17 & 17 & $0.55[0.29,1.03]$ & 0 & 0.06 \\
\hline \multicolumn{6}{|l|}{ 1st infusion infusion-related adverse events $*$} \\
\hline & 62 & 44 & $0.91[0.65,1.27]$ & 0 & 0.58 \\
\hline \multicolumn{6}{|l|}{ 2nd infusion infusion-related adverse events * } \\
\hline & 35 & 22 & $0.97[0.59,1.61]$ & 0 & 0.91 \\
\hline \multicolumn{6}{|l|}{ 3rd infusion infusion-related adverse events * } \\
\hline & 31 & 12 & $1.52[0.81,2.88]$ & 0 & 0.19 \\
\hline 4th infusion infusion-related adverse events $*$ & 31 & 6 & $2.95[1.26,6.90]$ & 0 & 0.01 \\
\hline
\end{tabular}

\section{Sensitivity analysis}

Sensitivity analysis is often used to evaluate the reliability of results. Ignoring the data of individual studies did not change the overall outcomes, which showed that outcomes were quite stable. Sensitivity analysis of the pooled data from the 13 observational studies was assessed. A significant change was not found in the outcomes, revealing that results of our observational studies are reliable.

\section{Net changes of efficacy parameters in RCTs}

A total of 241 patients received rituximab and 160 patients received a placebo in the two RCTs9,10, with 52 weeks as the end point. No heterogeneity was found between the 2 RCTs, and the fixed-effects model was applied. Relative to the placebo group, we observed a remarkable net increase of serum complement $\mathrm{C} 3$ in the rituximab group (WMDfixed $=7.67 \mathrm{mg} / \mathrm{dL}, 95 \%$ $\left.\mathrm{CI}=-0.16-15.50, \mathrm{I}^{2}=0 \%, \mathrm{P}=0.05\right)$, as shown in Fig. $2 \mathrm{~A}$. 


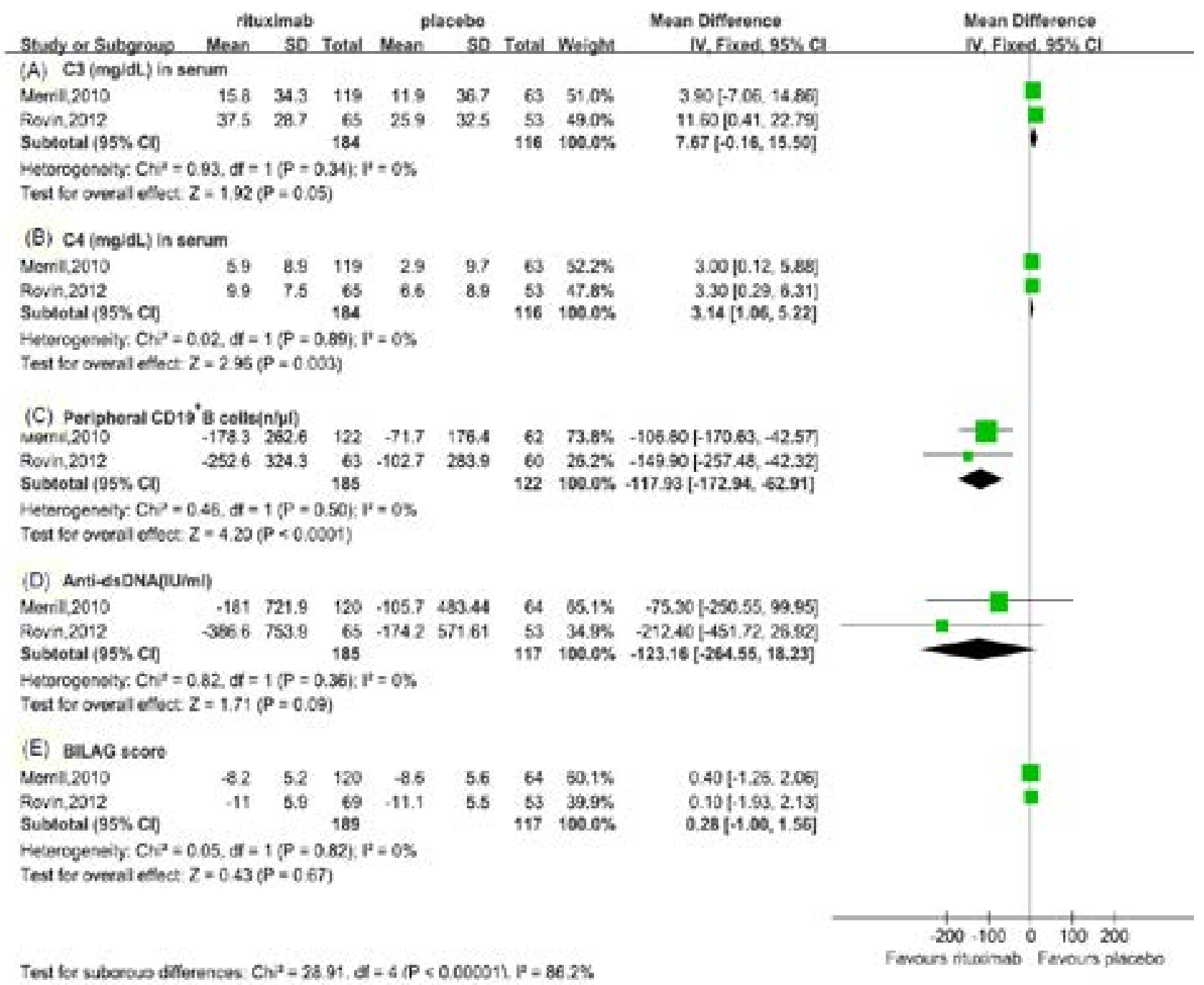

Fig.2 Efficacy parameters of rituximab $(1,000 \mathrm{mg})$ in two RCTs.

Compared to the placebo group, a significant increase in serum complement $\mathrm{C} 4$ was observed in the rituximab group (WMDfixed $=3.14 \mathrm{mg} / \mathrm{dL}, 95 \% \mathrm{CI}=1.06-5.22$, $\left.\mathrm{I}^{2}=0 \%, \mathrm{P}=0.003\right)$, as shown in Fig. 2B. A notable decrease in peripheral CD19 $9^{+} \mathrm{B}$ cells was observed in the rituximab group (WMDfixed $=-117.93 \mathrm{n} / \mu \mathrm{L}, 95 \% \mathrm{CI}=$ 172.94--62.91, $\mathrm{I}^{2}=0 \%, \mathrm{P}<0.0001$ ), as illustrated in Fig. 2C. Changes in serum anti-dsDNA antibodies were not significantly different between the rituximab and placebo groups (WMDfixed $=-123.16 \mathrm{I} \mathrm{U} / \mathrm{mL}, 95 \% \mathrm{CI}=$.
264.55-18.23, $\left.\mathrm{I}^{2}=0 \%, \mathrm{P}=0.09\right)$, asdepicted in Fig. 2D. Changes in the BILAG score did not differ between the rituximab and placebo groups (WMDfixed $=0.28$, 95\% $\left.\mathrm{CI}=-1.00-1.56, \mathrm{I}^{2}=0 \%, \mathrm{P}=0.67\right)$, as shown in Fig. $2 \mathrm{E}$. Clinical responses were assessed as the combination of complete and partial responses. The clinical responses were not significantly different between the rituximab and placebo groups (RRfixed $=1.14,95 \% \mathrm{CI}=0.88-1.48$, $\mathrm{I}^{2}=0 \%, \mathrm{P}=0.31$ ), as shown in Fig. 3 .

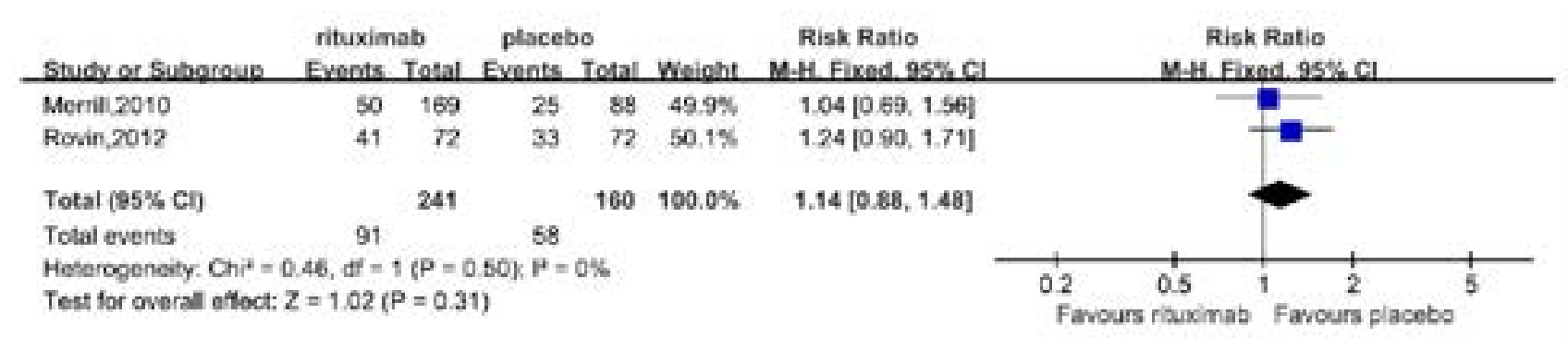

Fig.3 Clinical responses of rituximab $(1,000 \mathrm{mg})$ in two RCTs 


\section{Safety of rituximab in RCTs}

The safety outcomes of rituximab are summarized in Table 3. The occurrence and severity of adverse events(AEs) were classified according to the National Cancer Institute Common Toxicity Criteria for Adverse Events (version 3.0). We considered the following AEs: SAEs, death, infections, gastrointestinal disorders, infusion-related SAEs and infusion-related AEs over 52 weeks. The above-mentioned safety parameters were dichotomous variables, and no heterogeneity was found between the two RCTs $\left(\mathrm{I}^{2}=0 \%\right)$. The statistical analysis revealed no significant differences between the rituximab and placebo group, except for the occurrence ratio of the 4th rituximab infusion, where infusion-related AEs were significantly increased in the rituximab group (RRfixed $=2.95,95 \% \mathrm{CI}=1.26-6.90, \mathrm{I}^{2}=0 \%, \mathrm{P}=0.01$ ), as illustrated in Fig. 4J.

Table 3 Baseline characteristics of 13 observational studies

\begin{tabular}{|c|c|c|c|c|c|c|c|c|c|c|c|c|c|c|c|c|}
\hline No & Study & $\begin{array}{l}\text { Enrolled patients } \\
\text { characters }\end{array}$ & $\mathrm{n}$ & $\mathrm{T}_{\mathrm{c}}$ & $\begin{array}{c}\text { Female } \\
\text { sex } \\
(\%)\end{array}$ & Rituximab dose & Other treatment & $\begin{array}{c}\text { SLEDAI } \\
\text { score } \\
\text { meantSD }\end{array}$ & $\begin{array}{c}\text { BILAG } \\
\text { score } \\
\text { meantSD }\end{array}$ & $\begin{array}{l}\text { Anti-dSDNA } \\
\text { (IU/mL) } \\
\text { mean } \leq \mathrm{SD}\end{array}$ & $\begin{array}{c}\mathrm{C} 3 \\
(\mathrm{mg} / \mathrm{dL}) \\
\text { mean } \mathrm{SD}\end{array}$ & $\begin{array}{c}\mathrm{C} 4 \\
(\mathrm{mg} / \mathrm{dL}) \\
\text { mean } \mathrm{SD}\end{array}$ & $\begin{array}{c}\mathrm{CD19}{ }^{+} \mathrm{B} \\
\text { cells }(\mathrm{n} / \mathrm{LL}) \\
\text { mean } \mathrm{SD}\end{array}$ & $\begin{array}{c}\text { serum creatinine } \\
(\mu \operatorname{mol} \mathrm{L}) \\
\text { mean } \mathrm{SD}\end{array}$ & $\begin{array}{c}\text { 24-h urinary } \\
\text { protein(g/24h) } \\
\text { mean } \leqslant \mathrm{SD}\end{array}$ & $\begin{array}{c}\text { urinary protein- } \\
\text { creatinine } \\
\text { ratio }(\mathrm{g} / \mathrm{L})(\mu \mathrm{mol} / \mathrm{L}) \\
\text { mean } \mathrm{SD}\end{array}$ \\
\hline 1 & $\begin{array}{l}\text { Leandro } \\
\text { et al.(19) }\end{array}$ & $\begin{array}{l}\text { Patients failed conventional } \\
\text { immunosuppressive therapy }\end{array}$ & 24 & 24 & 91.7 & $\begin{array}{l}6 \text { patients } 2 \text { infusions } \\
\text { of } 500 \mathrm{mg}, 18 \text { patients } \\
2 \text { infusions of } 1000 \mathrm{mg} \\
\text { given } 2 \text { weeks apart }\end{array}$ & $\begin{array}{l}\text { Infusion CYC or } \\
\text { prednisolone, } \\
\text { continue } \\
\text { prednisolone and } \\
\text { HCQ }\end{array}$ & $\begin{array}{c}\text { Not } \\
\text { mentioned }\end{array}$ & $13.6 \pm 5.8$ & $270.3 \pm 251.7$ & $65.0 \pm 5.0$ & $\begin{array}{c}\text { Not } \\
\text { mentioned }\end{array}$ & $\begin{array}{c}\text { Not } \\
\text { mentioned }\end{array}$ & $\begin{array}{c}\text { Not } \\
\text { mentioned }\end{array}$ & $\begin{array}{c}\text { Not } \\
\text { mentioned }\end{array}$ & $417.6 \pm 103.0$ \\
\hline 2 & $\begin{array}{l}\text { Abitbol et } \\
\text { al.(20) }\end{array}$ & $\begin{array}{l}\text { Patients with severe SLE and } \\
\text { lupus nephririti age }<16 \\
\text { years }\end{array}$ & 18 & 24 & 88.9 & $\begin{array}{l}\text { The initial dose } \\
\text { was } 188 \\
\mathrm{mg} / \mathrm{m}^{2} \text {,subsequent } \\
\text { doses were } 375 \mathrm{mg} / \mathrm{m}^{2}\end{array}$ & $\begin{array}{l}\text { Low-dose } \\
\text { corticosteroids and } \\
\text { HCQ, maintenance } \\
\text { doses of MMF or }\end{array}$ & $\begin{array}{c}\text { Not } \\
\text { mentioned }\end{array}$ & $\begin{array}{l}\text { Not } \\
\text { mentione } \\
\text { d }\end{array}$ & $\begin{array}{c}1350.5 \pm 402 . \\
1 \\
\mathrm{mg} / \mathrm{dl}\end{array}$ & $61.4 \pm 8.4$ & $12.0 \pm 2.1$ & $243.0 \pm 223.0$ & $\begin{array}{c}1.2 \pm 0.4 \\
\mathrm{mg} / \mathrm{dl}\end{array}$ & $\begin{array}{c}\text { Not } \\
\text { mentioned }\end{array}$ & $\begin{array}{r}4.0 \pm 3.5 \\
(\mathrm{mg} / \mathrm{mg})\end{array}$ \\
\hline 3 & $\begin{array}{l}\text { Tamimot } \\
0 \text { et } \\
\text { al. }(21)\end{array}$ & $\begin{array}{l}\text { Refractory SLE failed to } \\
\text { corticosteroids and } \\
\text { immunosuppressive }\end{array}$ & 8 & 48 & 87.5 & $\begin{array}{l}100 \mathrm{mg} / \mathrm{m}^{2} \text { for } 3, \\
250 \mathrm{mg} / \mathrm{m}^{2} \text { for } 2, \\
375 \mathrm{mg} / \mathrm{m}^{2} \text { for } 3 \text { on } \\
\text { days } 1,8,15 \text { and } 22\end{array}$ & $\begin{array}{l}\text { A7 A A A } \\
\text { Predisolone 12.5- } \\
50.0 \mathrm{mg}, \\
\text { cyclosporine A } 75- \\
175 \mathrm{mg} \text { and }\end{array}$ & $17.6 \pm 10.2$ & $\begin{array}{c}\text { Not } \\
\text { mentione } \\
\text { d }\end{array}$ & $\begin{array}{c}\text { Not } \\
\text { mentioned }\end{array}$ & $\begin{array}{c}\text { Not } \\
\text { Mentioned }\end{array}$ & $\begin{array}{c}\text { Not } \\
\text { mentioned }\end{array}$ & $75.5 \pm 64.5$ & $\begin{array}{c}\text { Not } \\
\text { mentioned }\end{array}$ & $\begin{array}{c}\text { Not } \\
\text { mentioned }\end{array}$ & $\begin{array}{c}\text { Not } \\
\text { mentioned }\end{array}$ \\
\hline 4 & $\begin{array}{l}\text { Liet } \\
\text { al. (22) }\end{array}$ & $\begin{array}{l}\text { Patients with Class III or } \\
\text { intravenous (IV) lupus } \\
\text { nephritis }\end{array}$ & 19 & 24 & 89.5 & Infusion of 1000mg & $\begin{array}{l}\text { Nortinnetarmide } \\
\text { IV } \\
\text { methylprednisolone } \\
\text { 250mg, predinsolone } \\
\text { reduce from } 30 \text { to } 5 \\
\text { mgday, IV infusions } \\
\text { CYC 750mg for } 10\end{array}$ & $9.2 \pm 3.4$ & $\begin{array}{l}\text { Not } \\
\text { mentione } \\
\text { d }\end{array}$ & $693.5 \pm 345.0$ & $55.0 \pm 21.0$ & $14.0 \pm 9.0$ & $\begin{array}{c}\text { Not } \\
\text { mentioned }\end{array}$ & $118.2 \pm 71.2$ & $4.0 \pm 2.2$ & $\begin{array}{c}\text { Not } \\
\text { mentioned }\end{array}$ \\
\hline 5 & $\begin{array}{l}\text { Pepper et } \\
\text { al. (23) }\end{array}$ & $\begin{array}{l}\text { Patients with class III/IV/V } \\
\text { lupus nephritis }\end{array}$ & 18 & 48 & 83.3 & $\begin{array}{l}\text { Two infusions } \lg \text { on } \\
\text { days } 1 \text { and } 15\end{array}$ & $\begin{array}{l}\text { IV } \\
\text { methylprednisolone } \\
500 \mathrm{mg}, \\
\text { maintenance with } \\
\text { MMF 1 gdday }\end{array}$ & $\begin{array}{c}\text { Not } \\
\text { mentioned }\end{array}$ & $\begin{array}{l}\text { Not } \\
\text { mentione } \\
d\end{array}$ & $\begin{array}{c}\text { Not } \\
\text { mentioned }\end{array}$ & $\begin{array}{c}\text { Not } \\
\text { mentioned }\end{array}$ & $\begin{array}{c}\text { Not } \\
\text { mentioned }\end{array}$ & $\begin{array}{c}\text { Not } \\
\text { mentioned }\end{array}$ & $96.8 \pm 45.5$ & $\begin{array}{c}\text { Not } \\
\text { mentioned }\end{array}$ & $324.6 \pm 290.2$ \\
\hline 6 & $\begin{array}{l}\text { Ortega et } \\
\text { al. (24) }\end{array}$ & $\begin{array}{l}\text { Active SLE with severe } \\
\text { manifestations }\end{array}$ & 10 & 48 & 80.0 & IV infusions of $1 \mathrm{~g}$ & $\begin{array}{l}\text { Dexamethasone 8mg } \\
\text { on days land } 15\end{array}$ & $12.0 \pm 5.9$ & $\begin{array}{c}\text { Not } \\
\text { mentioned }\end{array}$ & $\begin{array}{c}\text { Not } \\
\text { mentioned }\end{array}$ & $\begin{array}{c}\text { Not } \\
\text { mentione } \\
\mathrm{d}\end{array}$ & $\begin{array}{c}\text { Not } \\
\text { mentioned }\end{array}$ & $175.4 \pm 50.8$ & $\begin{array}{c}\text { Not } \\
\text { mentioned }\end{array}$ & $\begin{array}{c}\text { Not } \\
\text { mentioned }\end{array}$ & $\begin{array}{c}\text { Not } \\
\text { Mentioned }\end{array}$ \\
\hline No & Study & $\begin{array}{l}\text { Enrolled patients } \\
\text { charaacters }\end{array}$ & $\mathrm{n}$ & $t_{\mathrm{c}}$ & $\begin{array}{c}\text { Female } \\
\text { sex } \\
(\%)\end{array}$ & Rituximab dose & Other treatment & $\begin{array}{c}\text { SLEDAI } \\
\text { score } \\
\text { mean } \mathrm{SD}\end{array}$ & $\begin{array}{c}\text { BILAG } \\
\text { score } \\
\text { mean } \pm \text { SD }\end{array}$ & $\begin{array}{c}\text { Anti- } \\
\text { dsDNA } \\
\text { (IU/ml) } \\
\text { mean } \leq S D\end{array}$ & $\begin{array}{c}\mathrm{C} 3 \\
(\mathrm{mg} / \mathrm{dl}) \\
\text { mean } \pm \mathrm{SD}\end{array}$ & $\begin{array}{c}C 4 \\
(\mathrm{mg} / \mathrm{dl}) \\
\text { meantSD }\end{array}$ & $\begin{array}{c}\mathrm{CD} 19^{+} \mathrm{B} \\
\text { cells }(\mathrm{n} / \mu \mathrm{l}) \\
\text { mean } \leqslant \mathrm{SD}\end{array}$ & $\begin{array}{c}\text { serum creatinine } \\
(\mu \mathrm{mol} / \mathrm{L}) \\
\text { mean } \mathrm{SD}\end{array}$ & $\begin{array}{c}\text { 24-h urinary } \\
\text { protein }(g / 24 h) \\
\text { mean } \leqslant \mathrm{SD}\end{array}$ & $\begin{array}{c}\text { urinary protein- } \\
\text { creatinine } \\
\text { ratio }(\mathrm{g} / \mathrm{L})(\mu \mathrm{mol} / \mathrm{L}) \\
\text { mean } \pm \mathrm{SD}\end{array}$ \\
\hline 7 & $\begin{array}{l}\text { Catapano } \\
\text { et al. (25) }\end{array}$ & Relapsing or refractory SLE & 31 & 48 & 90.3 & $\begin{array}{l}375 \mathrm{mg} / \mathrm{m}^{2} / \text { week } \times 4 \\
\text { infusions for } 16,1000 \\
\mathrm{mg} \times 2 \text { infusions for } \\
15 \text { patients }\end{array}$ & $\begin{array}{l}\text { IV intravenous CYC } \\
\text { 500mg and IV } \\
\text { methylprednisolone } \\
500-1000\end{array}$ & $\begin{array}{c}\text { Not } \\
\text { mentioned }\end{array}$ & $\begin{array}{c}\text { Not } \\
\text { mentioned }\end{array}$ & $\begin{array}{c}\text { Not } \\
\text { mentioned }\end{array}$ & $52.0 \pm 7.0$ & $8.0 \pm 1.0$ & $\begin{array}{c}\text { Not } \\
\text { mentioned }\end{array}$ & $86.8 \pm 17.2$ & $\begin{array}{c}\text { Not } \\
\text { mentioned }\end{array}$ & $\begin{array}{c}\text { Not } \\
\text { mentioned }\end{array}$ \\
\hline 8 & $\begin{array}{l}\text { Vitalet } \\
\text { al. (26) }\end{array}$ & Active severe SLE & 39 & 40 & $\begin{array}{c}\text { Not } \\
\text { mentione- } \\
d \mathrm{~d}\end{array}$ & $\begin{array}{l}1,000 \mathrm{mg} \text { on days } 1 \\
\text { and } 14\end{array}$ & $\begin{array}{l}\text { Infusions } \\
\text { methylpres } \\
\text { prednisolone 30- } \\
\text { 60mg on days1- } \\
\text { 14and background } \\
\text { immunosuppressants }\end{array}$ & & $4.0 \pm 4.3$ & $\begin{array}{c}\text { Not } \\
\text { mentioned }\end{array}$ & $\begin{array}{c}\text { Not } \\
\text { mentione } \\
d\end{array}$ & $\begin{array}{c}\text { Not } \\
\text { mentioned }\end{array}$ & $\begin{array}{c}\text { Not } \\
\text { mentioned }\end{array}$ & $\begin{array}{c}\text { Not } \\
\text { mentioned }\end{array}$ & $\begin{array}{c}\text { Not } \\
\text { mentioned }\end{array}$ & $\begin{array}{c}\text { Not } \\
\text { mentioned }\end{array}$ \\
\hline 9 & $\begin{array}{l}\text { Bang et } \\
\text { al. (27) }\end{array}$ & $\begin{array}{l}\text { Refractory SLE for } \\
\text { conventional therapy }\end{array}$ & 39 & 24 & 92.1 & $\begin{array}{l}2 \text { infusions } 500 \mathrm{mg} \text { for } \\
23,375 \mathrm{mg} / \mathrm{m}^{2} / \text { week } \\
\text { for } 5,2 \text { infusions } 1000 \\
\mathrm{mg} \text { for } 4\end{array}$ & $\begin{array}{l}\text { MMF for } 19 \text {, CYC } \\
\text { for } 17, \text { AZA for } 13 \text {, } \\
\text { cyclosporine for } 9\end{array}$ & $10.8 \pm 7.1$ & $\begin{array}{c}\text { Not } \\
\text { mentioned }\end{array}$ & $\begin{array}{c}\text { Not } \\
\text { mentioned }\end{array}$ & $70.9 \pm 27.9$ & $15.6 \pm 12.6$ & $\begin{array}{c}\text { Not } \\
\text { mentioned }\end{array}$ & $\begin{array}{c}\text { Not } \\
\text { mentioned }\end{array}$ & $\begin{array}{c}\text { Not } \\
\text { mentioned }\end{array}$ & $\begin{array}{c}\text { Not } \\
\text { Mentioned }\end{array}$ \\
\hline 10 & $\begin{array}{l}\text { Zhang et } \\
\text { al. (28) }\end{array}$ & $\begin{array}{l}\text { Refractory and severelupus } \\
\text { nephritis }\end{array}$ & 42 & 24 & 73.8 & 4 infusions $375 \mathrm{mg} / \mathrm{m}^{2}$ & $\begin{array}{l}\text { Methylprednisolone } \\
\text { 5000mgrdday, } \\
\text { prednisone } 0.6 \\
\text { mgkg daily for } 4 \\
\text { weeks, CTXX } 800 \mathrm{mg} \\
\text { at weeks } 1 \text { and } 3\end{array}$ & $14.9 \pm 3.5$ & $\begin{array}{l}\text { Not } \\
\text { mentione } \\
\text { d }\end{array}$ & $\begin{array}{c}\text { Not } \\
\text { mentioned }\end{array}$ & $35.0 \pm 19.0$ & $11.0 \pm 4.0$ & $\begin{array}{c}\text { Not } \\
\text { mentioned }\end{array}$ & $115.1 \pm 32.4$ & $4.8 \pm 1.9$ & $\begin{array}{c}\text { Not } \\
\text { mentioned }\end{array}$ \\
\hline 11 & $\begin{array}{l}\text { Qiu et al. } \\
\text { (29) }\end{array}$ & Active severe SLE & 27 & 64 & 81.5 & $\begin{array}{l}100 \mathrm{mg} / \text { week for } 4 \\
\text { weeks }\end{array}$ & $\begin{array}{l}\text { Methylprednisolone } \\
\text { infusions } 40 \mathrm{mg} \text { for } 4\end{array}$ & $19.0 \pm 10.0$ & $15.0 \pm 3.0$ & $\begin{array}{c}\text { Not } \\
\text { mentioned }\end{array}$ & $\begin{array}{c}\text { Not } \\
\text { mentione }\end{array}$ & $\begin{array}{c}\text { Not } \\
\text { mentioned }\end{array}$ & $\begin{array}{c}\text { Not } \\
\text { mentioned }\end{array}$ & $\begin{array}{c}\text { Not } \\
\text { mentioned }\end{array}$ & $\begin{array}{c}\text { Not } \\
\text { mentioned }\end{array}$ & $\begin{array}{c}\text { Not } \\
\text { mentioned }\end{array}$ \\
\hline 12 & $\begin{array}{l}\text { Liw et al. } \\
\text { (30) }\end{array}$ & Active SLE & 32 & 52 & 78.1 & $\begin{array}{l}100 \mathrm{mg} / \text { week for } \\
\text { 4weeks }\end{array}$ & $\begin{array}{l}\text { weeks } \\
\text { Methyprednisolone2 } \\
50 \text {-500 mgdday for } 3 \\
\text { days }\end{array}$ & $18.9 \pm 6.2$ & $16.3 \pm 4.1$ & $\begin{array}{c}\text { Not } \\
\text { mentioned }\end{array}$ & $\begin{array}{c}\text { d } \\
\text { Not } \\
\text { mentione } \\
d\end{array}$ & $\begin{array}{c}\text { Not } \\
\text { mentioned }\end{array}$ & $570 \pm 130$ & $\begin{array}{c}\text { Not } \\
\text { mentioned }\end{array}$ & $\begin{array}{c}\text { Not } \\
\text { mentioned }\end{array}$ & $\begin{array}{c}\text { Not } \\
\text { mentioned }\end{array}$ \\
\hline 13 & $\begin{array}{l}\text { Jiang et } \\
\text { al. (31) }\end{array}$ & Active SLE & 34 & 52 & 79.4 & $\begin{array}{l}100 \mathrm{mg} / \text { week for } \\
4 \text { weeks }\end{array}$ & $\begin{array}{l}\text { Methylprednisolone2 } \\
50-500 \mathrm{mg} \text { dday for } 3 \\
\text { days }\end{array}$ & 18. $9 \pm 6.2$ & $\begin{array}{l}16.27 \pm 4.0 \\
5\end{array}$ & $\begin{array}{c}\text { Not } \\
\text { mentioned }\end{array}$ & $\begin{array}{c}\text { Not } \\
\text { mentione } \\
\mathrm{d}\end{array}$ & $\begin{array}{c}\text { Not } \\
\text { mentioned }\end{array}$ & $\begin{array}{c}\text { Not } \\
\text { mentioned }\end{array}$ & $\begin{array}{c}\text { Not } \\
\text { mentioned }\end{array}$ & $\begin{array}{c}\text { Not } \\
\text { mentioned }\end{array}$ & $\begin{array}{c}\text { Not } \\
\text { mentioned }\end{array}$ \\
\hline
\end{tabular}

SLE: Systemic lupus erythematosus; SLEDAI: Systemic lupus erythematosus disease activity index; BILAG: British Isles Lupus As sessment Group index; CYC: cyclophosphamide; HCQ: hydroxychloroquine; MMF: mycophenolate mofetil; AZA: azathioprine. n: number enrolled; t: 
rituximah placehn

Rick Ratin

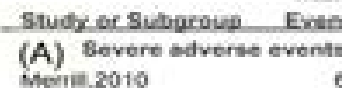

Rown, 2012

$\begin{array}{lrlll}64 & 169 & 32 & 60 & 13.2 \% \\ 24 & r 3 & 29 & n 1 & 9.2 \%\end{array}$

M. Hiatd. ass el

M. Fined - 95\% CI

Subtotal $[95 \% \mathrm{CI}$

$1.04[0.74,1.46]$

Total everts

es

$\begin{array}{llll}73 & 29 & 71 & 9.2 \%\end{array}$

$0.34[0.72,1.29]$

Heterogeneity: Chit = 0.85. of $=1(P=0.36 t P=0.5$

Test for overall effect $Z=0.42(P=0.67)$

(B) Death

Momil, 2010

Rovin 2012

Qubtotal cos\% co

4160

$15922.4 \%$

Total everts

27.

tas a 19

$0.4 \% 2.00[0.24,1 \mathrm{~s} .35]$

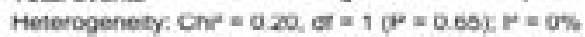

Test for overall effect: $Z=1,10(P=0.23)$

(C) Infections

Mernil. 2010

Rewin 2012

Subtotal [95\% CD]

$16 \quad 169$

jo

$242 \quad 14 \quad 71 \quad 44 \%$

20

$15910.6 \mathrm{~s}$

Total evonts

$\theta=1(p=0.24)+F=205$

Tesh for wrerall ellout $Z=1.23(P=0.10)$

(D) Gastrointestinal sisordera

Mecria, 2010

Rovin 2012

Subtotal (95\%, C])

8 160

Total everts

242

11

242

7

$60[0.22 .1 .50]$

0.49 [0.13. 1.27$]$

$0.55[0.25,1.22]$ 13

fest for overall eflect $Z=1.47\{P=0.14\}$

(E) Any infusion-related severe adverse events

Merrit, $2010 \quad 10$ 109 15

Rovin, 2012

Subtotal (95\% Ci)

173

$2 \quad 71 \quad 0.2 \%$

$0.6 \%$

$0.5 e[0.29,1.07]$

$0.49[0.05,5.24$

Total events $\quad 17 \quad 17 \quad 17$
Heterogeneity: Che $=0.01$, of $=1(P=0.92 x, P=0$.

Total events $\quad 17 \quad 17 \quad 17$
Heterogeneity: Che $=0.01, f^{\prime}=1(P=0.92 x+P=0$.

$1590.0 \%$

Test for overall efflect: $Z=1.86\langle P=0.06\}$

(F) amy infusion-related adverse events

$\begin{array}{llllll}\text { Rovin.2012 } & 75 & 73 & 20 & 71 & 0.29\end{array}$

Subtotal (205\% CD)

25.242

$159-23.24$

Futal evonts

99 63

Heterogeneity, Chr $=1.26$, af $=1, p=0.20), \mathrm{r}=20 \mathrm{k}$

test bor owerall efect: $Z=0.13(p=0.90)$

(G) First infusion infusion-retated adverse events

$\begin{array}{lllll}\text { Merrib, } 2010 & 46 & 169 & 26 & 88\end{array}$

Rovin. 2012

Subtotal $(95 \% \mathrm{Ci})$

1672

$18 \quad 72 \quad 5.6 \%$

$5.6 \%$

Total events

62 44

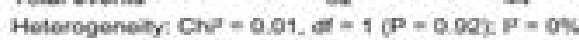

Test for coverall eflect: $Z=0.55$ (P $=0.58$ )

(H) Second infusion infusion-related adverse events

$\begin{array}{llllll}\text { Meerill, } 2010 & 29 & 165 & 14 & 65 & 5.6 \%\end{array}$

Rovin, 2012

Subtotal (9S\% Ci)

Total events

671 8 $71 \quad 2.5 \%$

$1.07[0.60,1.91]$

0.7510 .27 .2 .05

$0.97[0.59,1.61]$

183, 155

$1.02[0.70,1.31]$

$0.92|0.61,1.38|$ $0.89[0.49,1.60]$

$0.91[0.65 .1 .27)$

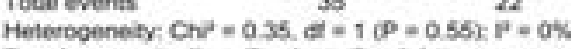

Test for overall ellect $Z=0.11$ (P $=0$.91)

(1) Third infusion infusion-rolated sdverse events

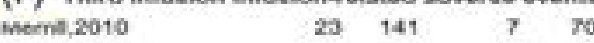

Puvin, 2012

Subtotal [35\% CI]

Total events

$2.9 \%$
$4.6 \%$

$1.60,[0.74,3.62]$

$1.34,0.40,3.80)$

$1.52[0.01,2.00]$

Heterogeneity Chit $=0.09$, 12

Tess for overall effect: $Z=1.30(P=0.19)$

(J) Fourth infusion infusion-related adverse events

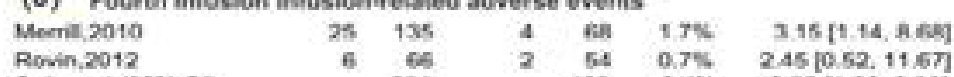

Subtotat (os\% CD $\quad 201 \quad 122 \quad 2.4 \% \quad 2.95[1.26,6.90]$

Total evonts 3s 6

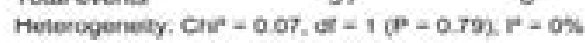

Test ace overall efect: $Z=2.49$ (p) $=0.01$ )

Total $(95 \% \mathrm{Cl})$

Total events 410

338

1518 100.0\%

$0.97[0.85,1.11]$

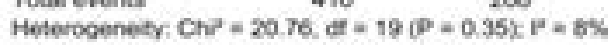

Test for coverall effect $Z=0.38$ ( $P=0.70)$

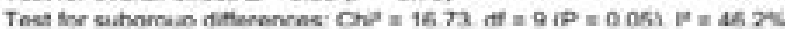

Fig.4 Safety of rituximab (1,000 mg) in two RCTs. 
Table 4 Quality assessment of included studies based on the Newcastle-Ottawa Scale

\begin{tabular}{|c|c|c|c|c|c|c|c|c|c|}
\hline Study & \multicolumn{8}{|c|}{ Quality evaluation ofobservational studies } & Scores \\
\hline $\begin{array}{l}\text { Leandro et } \\
\text { al. (19) }\end{array}$ & * & * & * & $*$ & * & * & * & * & 8 \\
\hline $\begin{array}{l}\text { Tamimoto et } \\
\text { al. (21) }\end{array}$ & et & $*$ & * & $*$ & * & $*$ & $*$ & $*$ & 8 \\
\hline Li et al. (22) & * & * & * & * & * & * & * & * & 8 \\
\hline $\begin{array}{l}\text { Pepper et } \\
\text { al. (23) }\end{array}$ & * & * & * & * & * & * & * & * & 8 \\
\hline $\begin{array}{l}\text { Ortegaet } \\
\text { al. (24) }\end{array}$ & * & * & * & * & * & * & * & $*$ & 8 \\
\hline $\begin{array}{l}\text { Zhang et } \\
\text { al. (28) }\end{array}$ & $*$ & $*$ & $*$ & $*$ & * & $*$ & $*$ & & 7 \\
\hline Qiu et al. (29) & * & * & * & * & * & * & * & * & 8 \\
\hline Liu et al. (30) & & * & * & * & * & * & * & & 6 \\
\hline $\begin{array}{l}\text { Jiang et } \\
\text { al. (31) }\end{array}$ & & * & * & * & * & * & * & & 6 \\
\hline
\end{tabular}

Evaluation of the efficacy of rituximab in observational studies

Observational studies data were grouped in this meta-analysis. Thirteen observational studies involving 341 patients (254 females) were included ${ }^{12-24}$. Summarized baseline characteristics of the included studies are shownin Table 3. Depending on whether patients received rituximab, patients were assigned to the baseline group and the "after rituximab" group. The baselinegroup was considered the control group, and the "af- ter rituximab" group was regarded as the intervention group.

In a total of 6 studies $^{14,17,21-24}, 153$ patients showed a net change in the SLE Disease Activity Index (SLEDAI) score. We adopted the random-effects model and observed that relative to baseline, rituximab useresulted in a significantly decreased in the "after rituximab" group (WMDrandom=-12.31, 95\% CI=-14.09--10.52, $\mathrm{I}^{2}=57 \%, \mathrm{P}<0.00001$, Fig. 5A). Additionally, moderate heterogeneity was found among studies $\left(\mathrm{I}^{2}=57 \%\right)$.

Table 5 The two most important patient outcomes are listed in the summary of findings table

\begin{tabular}{|c|c|c|c|c|c|c|}
\hline \multicolumn{7}{|c|}{$\begin{array}{l}\text { Rituximab Versus Placebo } \\
\text { Patient or population: patients with Systemic lupus erythematosus } \\
\text { Settings: in adult patients } \\
\text { Intervention: rituximab }\end{array}$} \\
\hline \multirow[b]{2}{*}{ Outcomes } & \multicolumn{2}{|c|}{$\begin{array}{l}\text { Illustrative comparative risks* }(95 \% \\
\text { CI) }\end{array}$} & \multirow[b]{2}{*}{$\begin{array}{l}\begin{array}{c}\text { Relative } \\
\text { effect }\end{array} \\
(95 \% \text { CI })\end{array}$} & \multirow[b]{2}{*}{$\begin{array}{l}\text { No of } \\
\text { Participants } \\
\text { (studies) }\end{array}$} & \multirow[b]{2}{*}{$\begin{array}{l}\text { Quality of } \\
\text { the } \\
\text { evidence } \\
\text { (GRADE) }\end{array}$} & \multirow[b]{2}{*}{ Comments } \\
\hline & Assumed Risk & $\begin{array}{l}\text { Corresponding risk } \\
\text { Death }\end{array}$ & & & & \\
\hline Death & 6 per 1000 & $\begin{array}{l}18 \text { per } 1000 \\
(3 \text { to } 102)\end{array}$ & $\begin{array}{l}\text { RR 2.86 } \\
(0.51 \text { to } \\
16.15)\end{array}$ & $\begin{array}{c}401 \\
\text { (2 studies) }\end{array}$ & $\begin{array}{l}\oplus \oplus \oplus \oplus \\
\text { high }\end{array}$ & \\
\hline $\begin{array}{l}\text { Severe adverse } \\
\quad \text { events }\end{array}$ & 384 per 1000 & $\begin{array}{l}361 \text { per } 1000 \\
(276 \text { to } 472)\end{array}$ & $\begin{array}{c}\text { RR } 0.94 \\
(0.72 \text { to } \\
1.23)\end{array}$ & $\begin{array}{c}401 \\
\text { (2 studies) }\end{array}$ & $\begin{array}{l}\oplus \oplus \oplus \oplus \\
\text { high }\end{array}$ & \\
\hline \multicolumn{7}{|c|}{$\begin{array}{l}\text { GRADE Working } \quad \text { Group } \\
\text { High quality: Further research is very unlikely to change our confidence in the estimate of effect. } \\
\text { Moderate quality: Further research is likely to have an important impact on our confidence in the estimate of effect and may change the } \\
\text { estimate. } \\
\text { Low quality: Further research is very likely to have an important impact on our confidence in the estimate of effect and is likely to change } \\
\text { the } \\
\text { Very low quality: We are very uncertain about the estimate. }\end{array}$} \\
\hline \multicolumn{7}{|c|}{$\begin{array}{l}\text { *The basis for the assumed risk (e.g. the median control group risk across studies) is provided in footnotes. The corresponding risk (and } \\
\text { its } 95 \% \text { confidence interval) is based on the assumed risk in the comparison group and the relative effect of the intervention (and its } 95 \% \\
\text { CI). } \\
\text { CI: Confidence interval; RR: Risk ratio. }\end{array}$} \\
\hline
\end{tabular}

In a total of 5 studies $^{12,19,22-24}, 156$ patients showed net changes in the BILAG score. The fixed-effects model was used, and compared to baseline, the BILAG score

African Health Sciences Vol 20 Issue 2, June, 2020 was obviously decreased in the "after rituximab" group (WMDfixed $=-9.72, \quad 95 \% \quad \mathrm{CI}=-10.42--9.01, \quad \mathrm{I}^{2}=0 \%$, $\mathrm{P}<0.00001$, Fig. 5C). Homogeneity was found among studies $\left(\mathrm{I}^{2}=0 \%\right)$. 


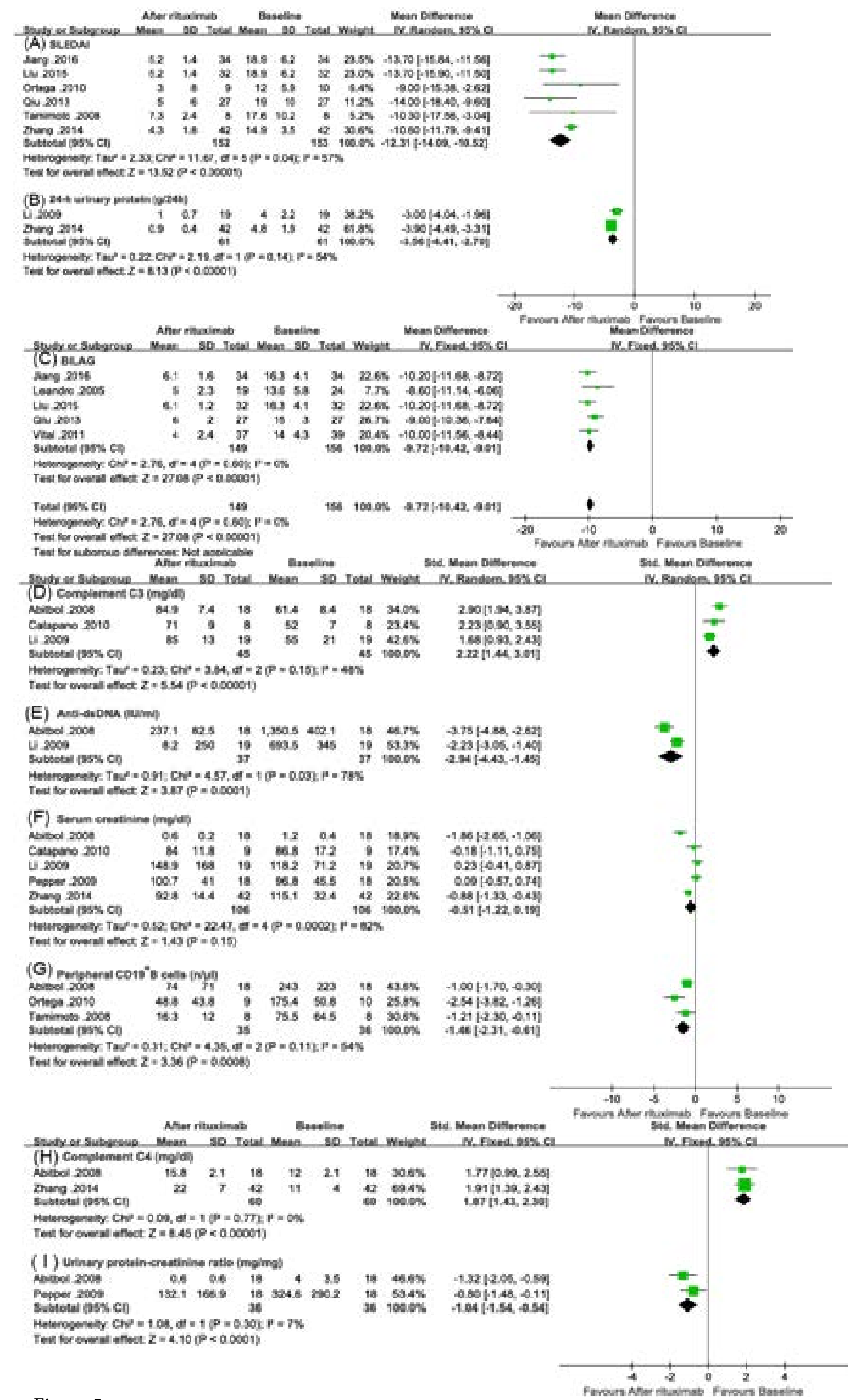

Figure 5 
In 3 studies, the serum complement C3 data 13, 15, 18 were reported for a total of 45 patients. We selected the random-effects model, and in contrast to baseline, a significant increase in complement C3 was observed in the "after rituximab" group (SMDrandom $=2.22,95 \%$ $\mathrm{CI}=1.44-3.01, \mathrm{I}^{2}=48 \%, \mathrm{P}<0.00001$, Fig. 5D). These studies exhibited moderate heterogeneity $\left(\mathrm{I}^{2}=48 \%\right)$.

Serum complement C4 data were reported in 2 studies 13, 21 including a total of 60 patients. We adopted the fixed-effects model and discovered that compared to baseline, a significant increase was observed in the "after rituximab" group (SMDfixed $=1.87,95 \% \mathrm{CI}=1.43$ 2.30, $\mathrm{I}^{2}=0 \%, \mathrm{P}<0.00001$, Fig. $\left.5 \mathrm{H}\right)$. No heterogeneity was observed between the two studies $\left(\mathrm{I}^{2}=0 \%\right)$.

Serum anti-dsDNA antibodies data were reported in 2 studies including a total of 37 patients 13, 15. The random-effects model was adopted, and a remarkable decrease in anti-dsDNA antibodies was observed in the "after rituximab" group compared to baseline(SMDran$\operatorname{dom}=-2.94,95 \% \mathrm{CI}=-4.43--1.45, \mathrm{I}^{2}=78 \%, \mathrm{P}=0.0001$, Fig.5E). High heterogeneity was observed between the two studies $\left(\mathrm{I}^{2}=78 \%\right)$. It was difficult to find the source of heterogeneity in the 2 studies.

Peripheral CD19+B-cell data were reported in 3 studies including a total of 36 patients13, 14, 17. The random-effects model was used, and a significant reduction in the "after rituximab" group was observed compared to baseline (SMDrandom $=-1.46,95 \% \mathrm{CI}=-2.31--0.61$, $\mathrm{I}^{2}=54 \%, \mathrm{P}=0.0008$, Fig. $\left.5 \mathrm{G}\right)$. These studies had moderate heterogeneity $(\mathrm{I} 2=54 \%)$.

Serum creatinine data were reported in 5 studies including a total of 106 patients13,15, 16, 18, 21. The random-effects model was adopted, and serum creatinine levels did not differ between the two groups (SMDrandom $=-0.51,95 \% \mathrm{CI}=-1.22-0.19, \mathrm{I}^{2}=82 \%, \mathrm{P}=0.15$, Fig. $5 \mathrm{~F})$. High heterogeneity was observed among studies $\left(\mathrm{I}^{2}=82 \%\right)$.

The 24-h urinary protein excretion data were reported in 2 studies including a total of 61 patients15, 21. We adopted the random-effects model and observed that 24-h urinary protein excretion was significantly decreased in the "after rituximab" group compared to baseline (WMDrandom $=-3.56$, 95\% CI $=-4.41--2.70$, $\mathrm{I}^{2}=54 \%, \mathrm{P}<0.00001$, Fig.5B). Medium heterogeneity was found between the two studies $\left(I^{2}=54 \%\right)$.

Urinary protein-creatinine ratio data were reported in 2 studies including 36 patients $^{13,16}$. The fixed-effects model was used, and a marked decrease in the urinary protein-creatinine ratio was observed in the "after rituximab" group compared to baseline (SMDrandom $=-1.04,95 \% \mathrm{CI}=-1.54-0.54, \quad \mathrm{I}^{2}=7 \%, \quad \mathrm{P}<0.0001$,
Fig.5I). Low heterogeneity was found between the two studies $\left(I^{2}=7 \%\right)$.

\section{Discussion}

In recent years, SLE patients have received many biotherapies, and these biological agents presented encouraging results. Rituximab is a biological agent that selectively targets $\mathrm{CD} 20^{+} \mathrm{B}$ cells. The earliest report of rituximab use in SLE patients was in $2001^{25}$. Favorable responses and satisfactory tolerance for rituximab use for refractory SLE patients were revealed in clinical trials. Particularly, these refractory patients had symptoms involving the renal, hematological and nervous systems $^{26,27}$. A good therapy should control SLE activity and prevent more organs from being impaired by severe or fatal outcomes.

Borba previously reported the following efficacy outcomes for rituximab: clinical response, BILAG C score, time-adjusted AUCMB of the BILAG score and modification in the SF-36 PCS. Considering these results, significant variations were not found between the rituximab and placebo groups ${ }^{11}$. Duxbury viewed rituximab can effectively control the activity of SLE in observational studies. Two RCTs did not display the benefit of rituximab by observing the complete response and the partial response rate ${ }^{28}$. Nevertheless, in our meta-analysis, both RCTs and observational studies showed that rituximab had satisfactory efficacy and safety results.

The BILAG and SLEDAI scores were used to assess the disease activity. These assessments consider clinical symptoms, physical signs, laboratory results and physician judgments. A lower score indicates that SLE is controlled and indirectly reflects the curative effect. We observed that rituximab and a placebo exhibited no differences regarding changes in BILAG scores in RCTs. Observational studies indicated that both BILAG and SLEDAI scores were remarkably reduced in the "after rituximab" group compared to baseline. The results of both BILAG and SLEDAI scores are consistent with the observational studies of Lan LAN ${ }^{29}$. The observational study outcomes suggest that rituximab is effective.

Higher anti-dsDNA antibodies and lower complement C3/C4 levels demonstrate the disease activity. We found a remarkable net increase in complement C3/ C4 in the rituximab group compared to the placebo group. Net changes of anti-dsDNA antibodies were similar between the rituximab and placebo groups, and the $\mathrm{P}$ value was close to $0.05(\mathrm{P}=0.09)$; additional RCTs may make the results significant. In contrast to baseline, complement $\mathrm{C} 3 / \mathrm{C} 4$ was significantly increased in 
the "after rituximab" group, and a remarkable decrease in anti-dsDNA antibodies was observed in the "after rituximab" group in observational studies. Despite that a distinct improvement of anti-dsDNA and complement $\mathrm{C} 3 / \mathrm{C} 4$ levels were not associated with Clinical outcomes, these changes correlated with the reduction of proteinuria in Lupus nephritis ${ }^{9}$. Fervenza observed that rituximab is superior to cyclosporine in maintaining complete or partial elimination of proteinura up to 24 months in membranous nephropathy ${ }^{30}$. The complement $\mathrm{C} 3 / \mathrm{C} 4$ results were reliable and showed that rituximab was efficacious in RCTs and observational studies, which suggested that the immune system was improved. The pathogenesis of SLE is attributed to the incidence of immune complexes that prompt supplementary pathway activation and complement consumption. Low complement C3/C4 levels are considered in the immunologic criteria of the Systemic Lupus International Collaborating Clinics (SLICC) when diagnosing and monitoring $\mathrm{SLE}^{31}$. These results indicated that rituximab can control disease activity and improve the immune system, but further investigations are still needed.

B-lymphocyte dysregulation is the focus of SLE pathogenesis, and B cells act as antigen-presenting cells that present autoantigens to T cells; $\mathrm{T}$ cells activate and produce cytokines. T cells and $\mathrm{B}$ cells stimulate each other, and autoantigen-specific B cells produce autoantibodies $^{3}$. This mechanism is complex; the role of $\mathrm{B}$ cells is not only restricted to producing antibodies ${ }^{32}$. Rituximab is a type of monoclonal antibody and targets CD20 on $B$ cells5, which exhausts B cells through different methods $^{33}$. CD19 ${ }^{+}$lymphocytes are B cells, and peripheral $\mathrm{CD} 19^{+} \mathrm{B}$ cells were significantly decreased in the rituximab group compared to the placebo group. Patients who received rituximab over 52 weeks maintained good B-cell depletion. The peripheral $\mathrm{CD} 19^{+} \mathrm{B}$ cells of the "after rituximab" group were remarkably decreased in observational studies. Both RCTs and observational studies demonstrated that rituximab can deplete peripheral CD $19^{+} \mathrm{B}$ cells, and these results are reliable. Sfikakis reported that refractory lupus nephritis patients who received rituximab attained $\mathrm{B}$-cell depletion and good clinical responses. The authors deduced that B-cell depletion was an effective therapy and that not only was an excessive production of autoantibodies avoided, but $\mathrm{B}$ cells were also hampered in presenting autoantigens to $T$ cells, and the potential activation of $\mathrm{T}$ helper cells was quickly reduced ${ }^{34}$. The B-cell depletion was similar between Sfikakis's results and our analysis.
The 24-h urinary protein excretion extremely important for reflecting the activity and severity of renal impairment in chronic kidney disease. The spot urinary protein-creatinine ratio(Up/Ucr) may be more efficient, reliable and time-saving to diagnose proteinuria in patients who are not pregnant ${ }^{35}$. The results of 24-h urinary protein excretion and the Up/Ucr were significantly decreased in the "after rituximab" group compared to baseline in the observational studies. Our 24-h urinary protein result was the same as that of Lan LAN in observational studies ${ }^{29}$. Our analysis shows that rituximab may be effective in patients with refractory and severe lupus nephritis.

The possible reasons of failure of rituximab therapy in randomized placebo-controlled trials are explained below. Firstly, as a background therapy (e.g. high-dose corticosteroids and full-dose MMF), immunosuppressive therapy may have masked an obvious clinical benefit of rituximab $\{\text { Ready V, } 2013 \# 41\}^{36}$. The composition of patients in the RCTs was different from that in the observational studies, as refractory patients were recruited in the observational studies but not enrolled in the RCTs. Moreover, factors of ethnic differences should be considered, with the African subgroup achieving a beneficial effect of rituximab in the RCTs. Secondly, we should pay more attention to background therapy. Ramos-Casals observed that the combination of rituximab and CYC may have synergistic effect and associated CYC with obvious benefits for complicated and refractory $\mathrm{SLE}^{37}$. Other views including Duxbury viewed that the number of patients in RCTs seemed too small (401 individuals ) to reflect superiority of rituximab over placebo $^{28}$.

The safety results of RCTs included SAEs, deaths, infections, gastrointestinal disorders, any infusion-related SAEs and infusion-related AEs. Previously mentioned studies showed no significant variation between the rituximab and placebo groups, except for the occurrence rate of the $4^{\text {th }}$ rituximab infusion, where infusion-related AEs were notably increased but did not affect the safety of rituximab applications. Our safety results are consistent with those of Borba, who concluded that rituximab is relatively safe for SLE patients ${ }^{11}$. Another purpose of using rituximab is a reduction in steroids dose, which avoids the side effects of steroids $^{38}$. There is a significant correlation between higher doses of rituximab and a decreased rate of infection. However, it cannot be excluded from the findings that infections led to the termination of rituximab treatment or lower $\operatorname{doses}^{39}$. Consequently, we recommend that rituximab 
is safe, but more high-quality long-term information is required.

The reviewed safety outcomes of rituximab has been presented in a table using the GRADE profiler (Table $5)^{9,10}$. The two most important safety outcomes of patient with SLE are displayed in the table.

Patients with fewer immunosuppressive drugs previously low titers of complement $\mathrm{C} 4$ and severe disease may respond better. This indicates that the ideal candidates for rituximab may be patients without obvious refractory process ${ }^{40-42}$. Relapses are related to increased damage. Thus, we should pay close attention to an appropriate balance between the dose and toxic risk of immunosuppressive drugs. As a maintenance treatment, Rituximab may be considered for refractory patients, for whom first-line immunosuppressive drugs are invalid. Moreover, there will be a high risk if these patients simply wait for symptomatic treatments after relapse ${ }^{42}$.

\section{Conclusion}

We observed that rituximab treatment may be promising, especially for severe and refractory SLE. However, further investigation and discussion are required.

\section{Methods}

We conducted a meta-analysis to estimate the efficacy and safety of rituximab treatment for SLE and followed the Cochrane Handbook ${ }^{43}$.

\section{Inclusion and exclusion criteria}

The inclusion criteria were as follows: (1) The SLE diagnosis satisfied the standards specified by the American College of Rheumatology ${ }^{44}$. (2) The trials included rituximab as an intervention treatment for SLE. (3) Placebo group as control group in RCTs. Baseline group when patients did not receive rituximab as control group in observational studies. (4) The study included efficacy and safety results, and the parameters of efficacy were the BILAG score, SLEDAI score, complement $\mathrm{C} 3 / \mathrm{C} 4$ levels, anti-dsDNA antibodies, peripheral CD19+B cells, serum creatinine, 24-h urinary protein and $\mathrm{Up} / \mathrm{Ucr}$. The safety results included the incidence of SAEs, deaths, infections, gastrointestinal disorders, infusion-related SAEs and infusion-related AEs. (5) Both RCT and observational studies that met the above conditions can be included in this study. Trials without clinical outcomes and articles that were merely obtainable as abstracts were excluded from the meta-analysis ${ }^{11}$. No language restrictions were implemented.

\section{Authors' contributions}

All authors made important contributions to this work.
Shanshan Wu and Yanhai Wang conceived and designed this research. Shanshan Wu and Jiaojiao Zhang searched articles and extracted data. Bo Han, Wanli Gao, Ning Zhang and Cheng Zhang verified and analyzed the data. All figures and tables were prepared by Yanhai Wang. Baishan Wang, Feng Yan and Zhijing Li wrote the manuscript. All authors reviewed and approved the manuscript.

\section{Funding}

This research was supported by the Natural Science Foundation of Liaoning Province of China (Grant No. 20170540595).

\section{Acknowledgments}

All authors are thankful to Drs. Merrill and Rovin for their kind help and for providing the raw data. We acknowledge the financial support of the Natural Science Foundation of Liaoning Province of China. We gratefully thank the American Journal Experts for their kind help.

\section{Conflicts of interest}

The authors declare that they have no potential conflicts of interest regarding the research, authorship, and publication of this article.

\section{References}

1. Achouak Achour, Amani Mankaï, Yosra Thabet, Wahiba Sakly, Fehmi Braham, Chedia Kechrid et al. Systemic lupus erythematosus in the elderly. Rheumatol Int 2012 May;32(5):1225-9.

2. David PD'Cruz, Munther A Khamashta, Graham RV Hughes. Systemic lupus erythematosus. Lancet $2007 \mathrm{Feb}$ 17;369(9561):587-96.

3. Anisur Rahman, David A. Isenberg. Systemic lupus erythematosus. N Engl J Med 2008 Feb 28;358(9):92939.

4. Ricard Cervera, Munther A. Khamashta, Josep Font, Gian Domenico Sebastiani, Antonio Gil, Paz Lavilla et al. Morbidity and mortality in systemic lupus erythematosus during a 10-year period: a comparison of early and late manifestations in a cohort of 1,000 patients. Medicine (Baltimore)2003 Sep;82(5):299-308.

5. Plosker GL, Figgitt DP. Rituximab: a review of its use in non-Hodgkin's lymphoma and chronic lymphocytic leukaemia. Drugs 2003;63(8):803-43.

6. R. John Looney, Jennifer H. Anolik, Debbie Campbell, Raymond E. Felgar, Faith Young, Lois J. Arend et al. B cell depletion as a novel treatment for systemic lupus erythematosus: a phase I/II dose-escalation trial of rituximab. Arthritis Rheum 2004 Aug;50(8):2580-9. 
7. D.Albert, S.Khan, J.Stansberry, S.Kolasinski, D.Tsai, M.Kamoun et al. A phase I trial of rituximab (anti-CD20) for treatment of systemic lupus erythematosus. Arthritis and Rheumatism 2003;48:3659.

8. Maria J. Leandro, Jonathan C. Edwards, Geraldine Cambridge, Michael R. Ehrenstein, Isenberg DA. An open study of B lymphocyte depletion in systemic lupus erythematosus. Arthritis Rheum 2002 Oct;46(10):2673-7. 9. Brad H.Rovin, Richard Furie, Kevin Latinis, R. John Looney, Fernando C.Fervenza, Jorge Sanchez-Guerrero et al. Efficacy and safety of rituximab in patients with active proliferative lupus nephritis: the Lupus Nephritis Assessment with Rituximab study. Arthritis Rheum 2012;64(4):1215-26.

10. Joan T. Merrill, C. Michael Neuwelt, Daniel J. Wallace, Joseph C. Shanahan, Kevin M.Latinis, James C.Oates et al. Efficacy and safety of rituximab in moderately-to-Severely active systemic lupus erythematosus: the randomized, double-blind, phase II/III systemic lupus erythematosus evaluation of rituximab trial. Arthritis Rheum 2010;62(1):222-33.

11. Helena Hiemisch Lobo Borba, Astrid Wiens, Thais Teles de Souza, Cassyano Januario Correr, Pontarolo R. Efficacy and safety of biologic therapies for systemic lupus erythematosus treatment: systematic review and meta-analysis. Bio Drugs 2014;28(2):211-28.

12. Deeks JJ, Higgins JPT, DG A. Chapter 9: analysing data and undertaking meta-analyses. . In Higgins J, Green S (eds)Cochrane Handbook for Systematic Reviews of Interventions Version 510(updated March 2011): The Cochrane Collaboration; 2011.

13. Hochberg MC. Updating the American College of Rheumatology revised criteria for the classification of systemic lupus erythematosus. Arthritis Rheum 1997;40(9):1725-25.

14. Nancy L Wilczynski, R Brian Haynes. Developing optimal search strategies for detecting clinically sound prognostic studies in MEDLINE: an analytic survey. BMC Med 2004;2(1):23.

15. Julian P. T.Higgins, Sally Green. Cochrane handbook for systematic reviews of interventions version5.1.0 updated March 2011. The Cochrane Collaboration 2011.

16. Alejandro R.Jadad, R. Andrew Moore, Dawn Carroll,Crispin Jenkinson, D. John M.Reynolds, David J.Gavaghan et al. Assessing the quality of reports of randomized clinical trials: is blinding necessary? Control Clin Trials 1996;17(1):1-12.

17. Andreas Stang. Critical evaluation of the NewcastleOttawa scale for the assessment of the quality of nonrandomized studies in meta-analyses. Eur J Epidemiol 2010 Sep;25(9):603-5.
18. Higgins J, Thompson SG. Quantifying heterogeneity in a meta-analysis. Stat Med 2002; 21:1539-58. 19. M. J. Leandro, G. Cambridge, J. C. Edwards, M. R. Ehrenstein, Isenberg DA. B-cell depletion in the treatment of patients with systemic lupus erythematosus: A longitudinal analysis of 24 patients. Rheumatology 2005;44(12):1542-45.

20. Obioma Nwobi, Carolyn L. Abitbol, Jayanthi Chandar, Wacharee Seeherunvong, Zilleruelo G. Rituximab therapy for juvenile-onset systemic lupus erythematosus. Pediatr Nephrol 2008 Mar;23(3):413-9.

21. Y. Tamimoto, T.Horiuchi, H.Tsukamoto, J.Otsuka, H.Mitoma, Y.Kimoto et al. A dose-escalation study of rituximab for treatment of systemic lupus erythematosusand Evans' syndrome: immunological analysis of Bcells, T cells and cytokines. Rheumatology (Oxford) 2008 Jun;47(6):821-7.

22. Edmund K. Li, Lai-Shan Tam, Tracy Y. Zhu, Martin Li, Catherine L. Kwok, Tena K. Li et al. Is combination rituximab with cyclophosphamide better than rituximab alone in the treatment of lupus nephritis? Rheumatology (oxford, england) serial on the Internet. 2009; 48(8): Available from: http:// onlinelibrary.wiley.com/o/ cochrane/clcentral/articles/916/CN-00722916/frame. html.

23. Ruth Pepper, Megan Griffith, Chris Kirwan, Jeremy Levy, David Taube, Charles Pusey et al. Rituximab is an effective treatment for lupus nephritis and allows a reduction in maintenance steroids. Nephrol Dial Transplant 2009 Dec;24(12):3717-23.

24. Lilia Andrade-Ortega, Fedra Irazoque-Palazuelos, Ricardo López-Villanueva, Yaneth Barragán-Navarro, Fernando Bourget-Pietrasanta, Maria de los Ángeles Díaz-Ceballos et al. Efficacy of rituximab versus cyclophosphamide in lupus patients with severe manifestations. A randomized and multicenter study. Reumatol Clin 2010 Sep-Oct;6(5):250-5.

25. Fausta Catapano, Afzal N. Chaudhry, Rachel B. Jones, Kenneth G.C. Smith, Jayne DW. Long-term efficacy and safety of rituximab in refractory and relapsing systemic lupus erythematosus. Nephrol Dial Transplant 2010 Nov;25(11):3586-92.

26. Edward M. Vital, Shouvik Dass, Maya H. Buch, Karen Henshaw, Colin T. Pease, Michael F. Martin et al. B cell biomarkers of rituximab responses in systemic lupus erythematosus. Arthritis Rheum 2011 Oct;63(10):303847.

27. So-Young Bang, Chang Keun Lee, Young Mo Kang, Hyoun-Ah Kim, Chang-Hee Suh, Won Tae Chung et al. Multicenter retrospective analysis of the effectiveness and safety of rituximab in Korean patients with refrac- 
tory systemic lupus erythematosus. Autoimmune Diseases 2012;1(1):1-6.

28. Jin Zhang, Zhanzheng Zhao, $\mathrm{Hu} \mathrm{X}$. Effect of Ritux-imab on Serum Levels of Anti-C1q and Antineutrophil Cytoplasmic Autoantibodies in Refractory Severe Lupus Nephritis. Cell Biochemistry and Biophysics Serial on the Internet 2014; 72(1): Available from: http://onlineli-brary.wiley.com/o/cochrane/clcentral/ articles/906/CN-01260906/frame.html.

29. Minli qiu, Ou jin, Linkai fang, Jieruo gu. Analysis of the Effect of Small Dose of Rituximab in the'Treatment of Systemic Lupus ErythematosusArticle in Chinese. China \& Foreign Medical Treatment 2013(25):21-22.

30. Liu X. Analysis of small dose of rituximab in the treatment of Systemic lupus erythematosusArticle in Chinese. China \& Foreign Medical Treatment 2015(13):14243.

31. Hua jiang, Yiying zhang, Mingrui du. Efficacy of low dose rituximab in the treatment of systemic lupus erythematosusArticle in Chinese. Chinese Hospital Pharmacy Journal 2016(36):1.

32. F. Petschner, U. A.Walker, A.Schmitt-Graff, M.Uhl, H. H.Peter. "Catastrophic systemic lupus erythematosus"with Rosai-Dorfman sinus histiocytosis. Successful treatment with anti-CD20/rutuximab. Dtsch Med Wochenschr 2001 Sep14;126(37):998-1001.

33. Sailler L. Rituximab off label use for difficult to treat auto-immune diseases: reappraisal of benefits and risks. Clinical Reviews in Allergy \& Immunology 2008 Feb;34(1):103-10.

34. M Ramos-Casals, MJ Soto, MJ Cuadrado, M. A.Khamashta. Rituximab in systemic lupus erythematosus:A systematic review of off-label use in 188 cases. Lupus 2009 Aug;18(9):767-76.

35. B Duxbury, C Combescure, Chizzolini. C. Rituximab in systemic lupus erythematosus: an updated systematic review and meta-analysis. Lupus 2013; 22(14):1489-503. 36. Lan Lan, Fei Han, Jiang-hua Chen. Efficacy and safety of rituximab therapy for systemic lupus erythematosus:a systematic review and meta-analysis. Journal of Zhejiang University-SCIENCE B 2012;13(9):731-44. 37. F.C. Fervenza, G.B. Appel, S.J. Barbour, B.H. Rovin, R.A. Lafayette, N. Aslam et al. Rituximab or Cyclo-sporine in the Treatment of Membranous Nephropa-thy. The New England Journal of Medicine 2019;381(1):36-46.

38. Michelle Petri. Systemic Lupus International CollaboratingClinic (SLICC). SLICC revision of the ACR classification criteria for SLE. Arthritis Rheum 2009;60(Suppl 10):895.

39. Jennifer L.Huggins, I.Brunner H. Targeting B cells in the treatment of childhood-onset systemic lupus er- ythematosus. J Pediatr 2006 May;148(5):571-3.

40. Ronald P Taylor, Lindorfer MA. Drug insight: the mechanism of action of rituximab in autoimmune disease--the immune complex decoy hypothesis. Nat Clin Pract Rheumatol 2007 Feb;3(2):86-95.

41. P. P.Sfikakis, J. N.Boletis, S.Lionaki, V.Vigklis, K. G.Fragiadaki, A.Iniotaki et al. Remission of proliferative lupus nephritis following $\mathrm{B}$ cell depletion therapy is preceded by down-regulation of the $\mathrm{T}$ cell costimulatory molecule C D40 ligand: An open-label trial. Arthritis Rheum 2005;52(2):501-13.

42. Jay M. Ginsberg, Bruce S. Chang, Richard A. Matarese,Serafino Garella. Use of single voided urine samples to estimate quantitative proteinuria. $N$ Engl J Med 1983 Dec 22;309(25):1543-6.

43. Ready V, Jayne D, Close D, D. I. B-cell depletion in SLE:Clinical and trial experience with rituximab and ocrelizumab and implications for study design. Arthritis Res Ther 2013;15(Suppl 1):S2.

44. Manuel Ramos-Casals, Candido Díaz-Lagares, Khamashta MA. Rituximab and lupus: good in real life, bad in controlled trials. Comment on the article by $\mathrm{Lu}$ et al. Arthritis Rheum 2009;61(9):1281-82.

45. Yoshiya Tanaka, Tsutomu Takeuchi, Nobuyuki Miyasaka, Takayuki Sumida, Tsuneyo Mimori, Takao Koike et al. Efficacy and safety of rituximab in Japanese patients with systemic lupus erythematosus including lupus nephritis who are refractory to conventional therapy. Mod Rheumatol 2016;26(1):80-6.

46. Hans-Peter Tony, Gerd Burmester, Hendrik Schulze-Koops, Mathias Grunke, Joerg Henes, Ina Kötter et al. Safety and clinical outcomes of rituximab therapy in patients with different autoimmune diseases: experience from a national registry (GRAID). Arthritis Research \&Therapy 2011;13(3):R75.

47. Fernandez-Nebro A, de la Fuente JL, Carreno L, Izquierdo MG, Tomero E, Rua-Figueroa I et al. Multicenter longitudinal study of B-lymphocyte depletion in refractory systemic lupus erythematosus: the LESIMAB study. Lupus 2012;21(10):1063-76.

48. Md Yusof MY, Shaw D, El-Sherbiny YM, Dunn E, Rawstron AC, Emery P et al. Predicting and managing primary and secondary non-response to rituximab using B-cell biomarkers in systemic lupus erythematosus. Ann Rheum Dis 2017;76(11):1829-36.

49. Matthias A. Cassia, Federico Alberici, Rachel B. Jones, Rona M.Smith, Giovanni Casazza, Maria L. Urban et al. Rituximab as Maintenance Treatment for Systemic Lupus Erythematosus: A Multicenter Observa-tional Study of 147 Patients. Arthritis Rheumatol 2019 May 18. 